\title{
Estudio comparativo del comportamiento de los contadores me- cánicos frente a los contadores de ultrasonidos y caudalímetros electromagnéticos
}

Madurga del Cura, Cristina1 ${ }^{1}$, Cervantes Díaz-Toledo, Alfonso², Muñoz Sopeña, Daniel², Rodríguez Fernández, Diego $^{2}$, Sánchez de Ribera González, Alejandro², Zazo Salinero, Teresa²

1 Ingeniero Agrónomo, TRAGSA - CENTER, cmadurga@tragsa.es

2 Equipo Técnico del CENTER

Resumen: Tradicionalmente se han instalado contadores mecánicos a pie de parcela, fundamentalmente por tema económico. Sin embargo, los nuevos materiales y los nuevos métodos de fabricación hacen que las nuevas tecnologías sean más accesibles, permitiendo la utilización de caudalímetros electromagnéticos y de ultrasonidos en redes de riego a pie de parcela.

El objetivo de este estudio es comparar el comportamiento de contadores mecánicos, caudalímetros electromagnéticos y de ultrasonidos bajo distintas condiciones de funcionamiento y configuraciones de montaje, así como su durabilidad siguiendo el procedimiento descrito en la norma UNE-ISO 16399 en su apartado 7.7.2 "Ensayo de resistencia a las partículas sólidas", incluido en la legislación española para contadores de riego (ICT/155/2020) que permite conocer la idoneidad o no de un equipo como contador de agua de riego al ser ensayados en condiciones reales de funcionamiento y con agua no limpia.

Para la realización de este estudio se va a analizar el comportamiento de los siguientes equipos:

- Contador mecánico tipo Woltman:

○ Eje horizontal

- Eje vertical

- Contador ultrasonidos: 2 modelos

- Caudalímetro electromagnético: 1 modelo

Todos los equipos serán DN80 con el objetivo de facilitar su comparativa.

Para cada uno de los equipos se obtendrá la curva de error bajo diferentes condiciones de funcionamiento, llevando a cabo los ensayos en el Laboratorio Central para Ensayo de Materiales y Equipos de Riego del CENTER.

Los resultados obtenidos servirán de orientación a promotores y usuarios a la hora de elegir el contador que más se adecúe a sus necesidades reales de funcionamiento.

Palabras clave: contadores; caudalímetros electromagnéticos; ultrasonidos; legislación contadores; laboratorio; ensayos; CENTER. 


\section{Comparative study of the behavior of mechanical meters com- pared to ultrasonic meters and electromagnetic flowmeters} Madurga del Cura, Cristina ${ }^{1}$, Cervantes Díaz-Toledo, Alfonso ${ }^{2}$, Muñoz Sopeña, Daniel $^{2}$, Rodríguez Fernández,
Diego $^{2}$, Sánchez de Ribera González, Alejandro², Zazo Salinero, Teresa ${ }^{2}$

1 Agricultural Engineer, TRAGSA - CENTER, cmadurga@tragsa.es

2 CENTER Technical Team

Abstract: Traditionally, mechanical meters have been installed on farm, mainly for economic reasons. However, new materials and new manufacturing methods make new technologies more accessible, allowing the use of electromagnetic flowmeters and ultrasonic meters in irritation networks with small diameters.

The aim of this study is to compare the behaviour of mechanical meters, ultrasonic meters and electromagnetic flow meters under different operating conditions and mounting configurations, as well as their durability following the procedure described in the standard UNE-ISO 16399, in its clause 7.2.2 "Resistance to solid particles test", included in the Spanish legislation for irrigation meters (ICT/155/2020). The result of this test allows knowing the suitability or not of a device as an irrigation water meter when tested under operating conditions and with not clean water.

To carry out this study, the behaviour of the following equipment is been analysed:

- Mechanical meter.

- Woltman horizontal axis.

- Woltman vertical axis

- Ultrasonic meter: 2 models

- Electromagnetic flowmeter: 1 model

All the equipment is DN80 in order to facilitate their comparison.

For each equipment, the error - curve is obtained under different operating conditions, carrying out the tests in the Central Laboratory for Testing Materials and Irrigation Equipment in the National Centre for Irrigation Technology (CENTER).

Keywords: electromagnetic flowmeter; ultrasonic meter; legislation for irrigation meters; laboratory; tests; CENTER 


\section{Introducción}

Los contadores de riego están regulados por la Orden ICT/155/2020 que establece los requisitos que deben cumplir estos equipos para poder ser comercializados e instalados en una red de riego, así como cuando ya están en funcionamiento.

En la actualidad, la mayor parte de los fabricantes de contadores los venden indistintamente para redes de agua limpia o para riego. Sin embargo, la calidad del agua para uso residencial, comercial o industria ligera difiere mucho de la del agua de riego.

Por ello, cuando en el subcomité de ISO TC/23/SC18 "Irrigation and drainage equipment and systems" se decidió redactar una norma que estableciera los requisitos mínimos de calidad que debería tener un contador para riego, desde el CENTER que lideraba este proyecto, se propuso la inclusión de un ensayo que permitiera diferenciar los contadores según su uso en función de su resistencia a las partículas sólidas. Esta norma es la ISO 16399:2014 que se adoptó como norma española en 2016 (UNE-ISO 16399:2016).

Este ensayo se ha incluido dentro de la legislación como obligatorio para los contadores de riego.

En estos momentos, la norma ISO 16399 está en proceso de revisión y es por ello que en el CENTER se están llevando a cabo numerosos ensayos con diferentes modelos y tecnologías de contadores, con el objetivo de conocer si los requisitos establecidos en la norma, relativos a la resistencia a las partículas sólidas, son adecuados o no para los equipos que hay actualmente en el mercado.

\section{Materiales y métodos}

\subsection{Equipos ensayados}

En estos momentos se están llevando a cabo un gran número de pruebas en diversos contadores de distintos modelos y tecnologías.

Las características de los equipos ensayados hasta ahora son:

Tabla 1: Contadores ensayados. Características.

\begin{tabular}{l|c|c}
\hline Tipo de contador & $\begin{array}{c}\text { Caudal permanente } \\
\left(\mathbf{Q}_{3}\right) \mathbf{~ m}^{\mathbf{3}} / \mathbf{h}\end{array}$ & $\begin{array}{c}\text { Ratio } \\
\left(\mathbf{R}=\mathbf{Q}_{3} / \mathbf{Q}_{1}\right)\end{array}$ \\
\hline Contador Woltman Eje horizontal & 100 & 200 \\
\hline Contador Woltman Eje vertical & 63 & 50 \\
\hline Contador ultrasonidos Modelo 1 & 63 & 125 \\
\hline Contador ultrasonidos Modelo 2 & 63 & 500 \\
\hline
\end{tabular}

Para cada modelo de contador se ha ensayado un mínimo de dos muestras.

Se puede observar que, además de la distinta tipología, el rango de caudales de trabajo de los equipos es completamente diferente, trabajando algunos de ellos a caudales muy bajos que no son habituales en agricultura, pero sí en agua limpia. 
 CARTAGENA 2021}

\subsection{Ensayo de resistencia a las partículas sólidas}

Este ensayo, recogido en las normas ISO 16399 e UNE-ISO 16399, permite comprobar la idoneidad o no de un contador a ser utilizado en una red de riego al someterlo al paso de agua con partículas en suspensión durante un número elevado de horas.

Aunque en el agua de riego se pueden encontrar diferentes tipos de partículas, el estandarizar este ensayo, hace que los resultados de diferentes equipos sean comparables.

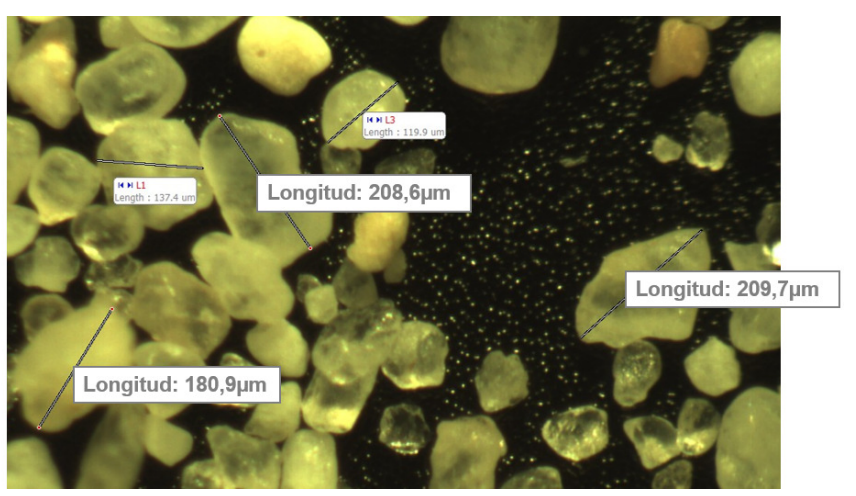

Figura 1: Partículas.

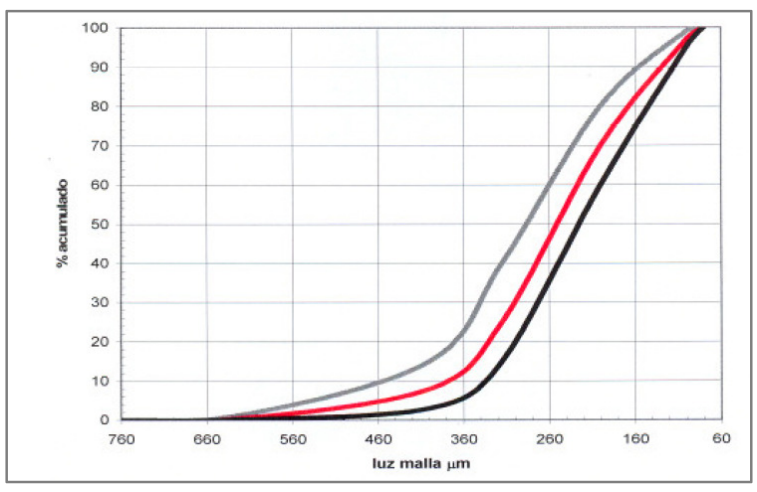

Figura 2: Distribución granulométrica.

El esquema del ensayo se muestra a continuación:

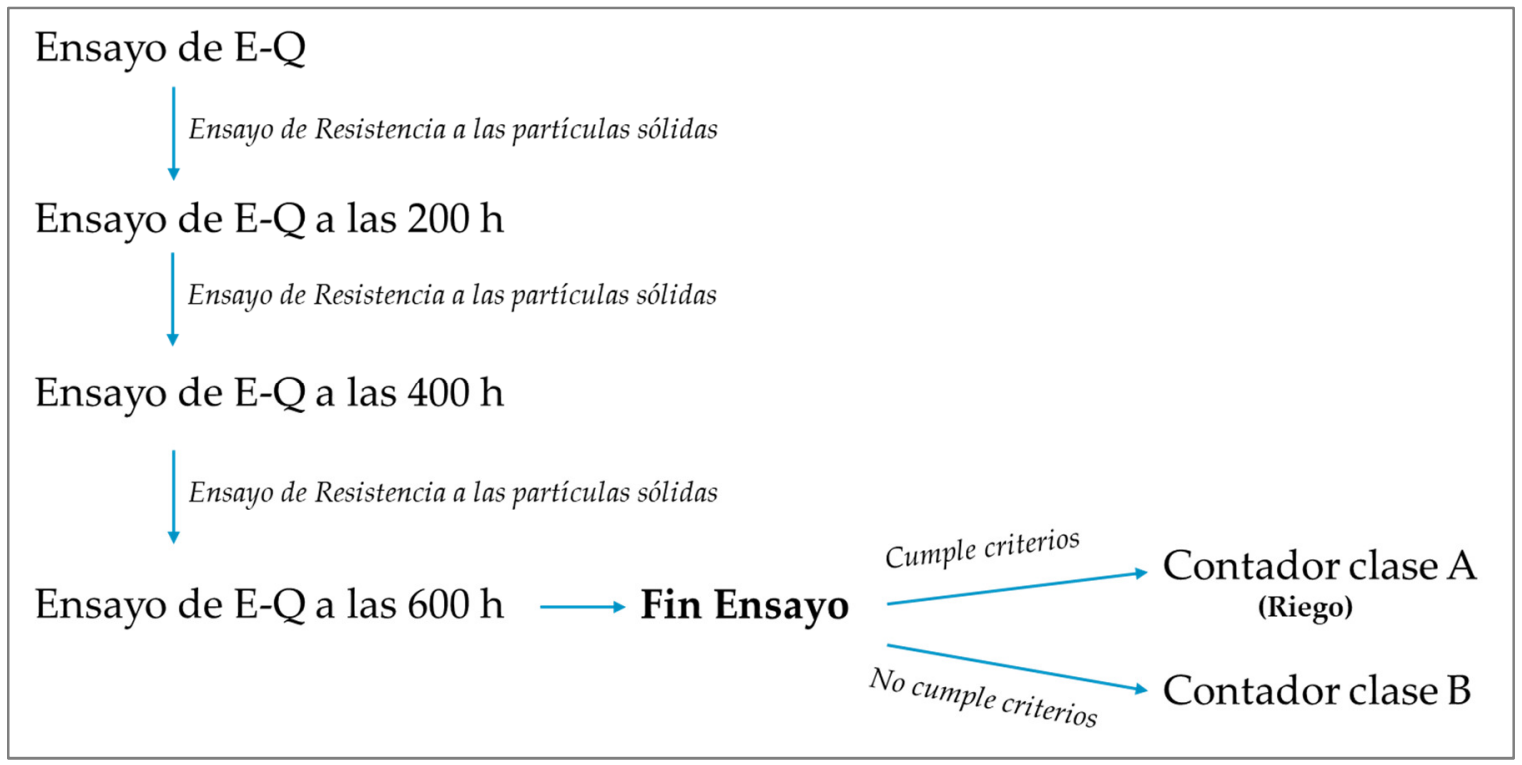

Figura 3: Esquema ensayo resistencia a las partículas sólidas.

Antes y después de cada una de las fases del ensayo de resistencia a las partículas sólidas se ha llevado a cabo la determinación de los errores (de indicación), es decir, la obtención de la curva de error en función del caudal inicial para los siguientes caudales de ensayo: 


\section{Congreso Nacional de Riegos CARTAGENA 2021}

Tabla 2: Determinación de errores (de indicación). Caudales de ensayo.

\begin{tabular}{c}
\hline ISO 16399/UNE-ISO 16399 \\
\hline $\mathrm{Q}_{1}-1,1 \mathrm{Q}_{1}$ \\
\hline $\mathrm{Q}_{2}-1,1 \mathrm{Q}_{2}$ \\
\hline $0,33\left(\mathrm{Q}_{2}+\mathrm{Q}_{3}\right)-0,37\left(\mathrm{Q}_{2}+\mathrm{Q}_{3}\right)$ \\
\hline $0,67\left(\mathrm{Q}_{2}+\mathrm{Q}_{3}\right)-0,74\left(\mathrm{Q}_{2}+\mathrm{Q}_{3}\right)$ \\
\hline $0,9 \mathrm{Q}_{3}-\mathrm{Q}_{3}$ \\
\hline $0,95 \mathrm{Q}_{4}-\mathrm{Q}_{4}$ \\
\hline
\end{tabular}

Los resultados obtenidos en esta prueba han de compararse con los errores máximos permitidos por la normativa y la legislación vigente que son:

$$
\begin{aligned}
& -\mathrm{Q}_{1} \leq \mathrm{Q}<\mathrm{Q}_{2}, \mathrm{EMP} \leq 5 \% \\
& -\mathrm{Q}_{2} \leq \mathrm{Q} \leq \mathrm{Q}_{4}, \mathrm{EMP} \leq 2 \%
\end{aligned}
$$

El error se expresa como porcentaje y equivale a:

$$
\varepsilon=\frac{\left(V_{i}-V_{a}\right)}{V_{a}} \times 100(\%)
$$

Siendo:

$$
\begin{aligned}
& \mathrm{Vi}=\text { Volumen indicado } \\
& \mathrm{Va}=\text { Volumen real }
\end{aligned}
$$

En el caso del banco del Laboratorio del CENTER, el volumen real es el indicado por las diferentes básculas, asegurando una precisión en la lectura mucho más alta que un contador o un caudalímetro patrón.

Al finalizar las $600 \mathrm{~h}$ del ensayo de resistencia a las partículas sólidas, los criterios de aceptación para la curva de error - caudal son los que se indican a continuación:

a) La variación en la curva de error no debe superar:

- $\pm 3 \%$ para caudales de la zona inferior $\left(\mathrm{Q}_{1} \leq \mathrm{Q}<\mathrm{Q}_{2}\right)$; $\mathrm{y}$

- $\pm 1,5 \%$ para caudales de la zona superior $\left(\mathrm{Q}_{2} \leq \mathrm{Q} \leq \mathrm{Q}_{4}\right)$.

Con el fin de determinar estos requisitos, se deben aplicar los valores medios de los errores en cada caudal de agua:

b) Las curvas de error no deben superar un límite de error máximo de:

- $\pm 6 \%$ para caudales de la zona inferior $\left(\mathrm{Q}_{1} \leq \mathrm{Q}<\mathrm{Q}_{2}\right)$; $\mathrm{y}$

- $\pm 2,5 \%$ para caudales de la zona superior $\left(\mathrm{Q}_{2} \leq \mathrm{Q} \leq \mathrm{Q}_{4}\right)$

\subsection{Laboratorio Central para Ensayo de Materiales y Equipos de Riego}

El Laboratorio Central para Ensayo de Materiales y Equipos de Riego, ubicado en el Centro Nacional de Tecnología de Regadíos (CENTER), órgano dependiente del Ministerio de Agricultura, Pesca y Alimentación (MAPA), dispone, entre otros, de un banco de ensayo de contadores con capacidad para realizar el ensayo de determinación de los errores (de indicación), así como un banco de durabilidad 
que permite estudiar el desgaste de los equipos con el paso del tiempo tanto con agua limpia como con agua con partículas en suspensión.

Para llevar a cabo el ensayo de resistencia a las partículas sólidas y asegurar una concentración homogénea, se dispone de cuatro agitadores que mantienen en suspensión dichas partículas.
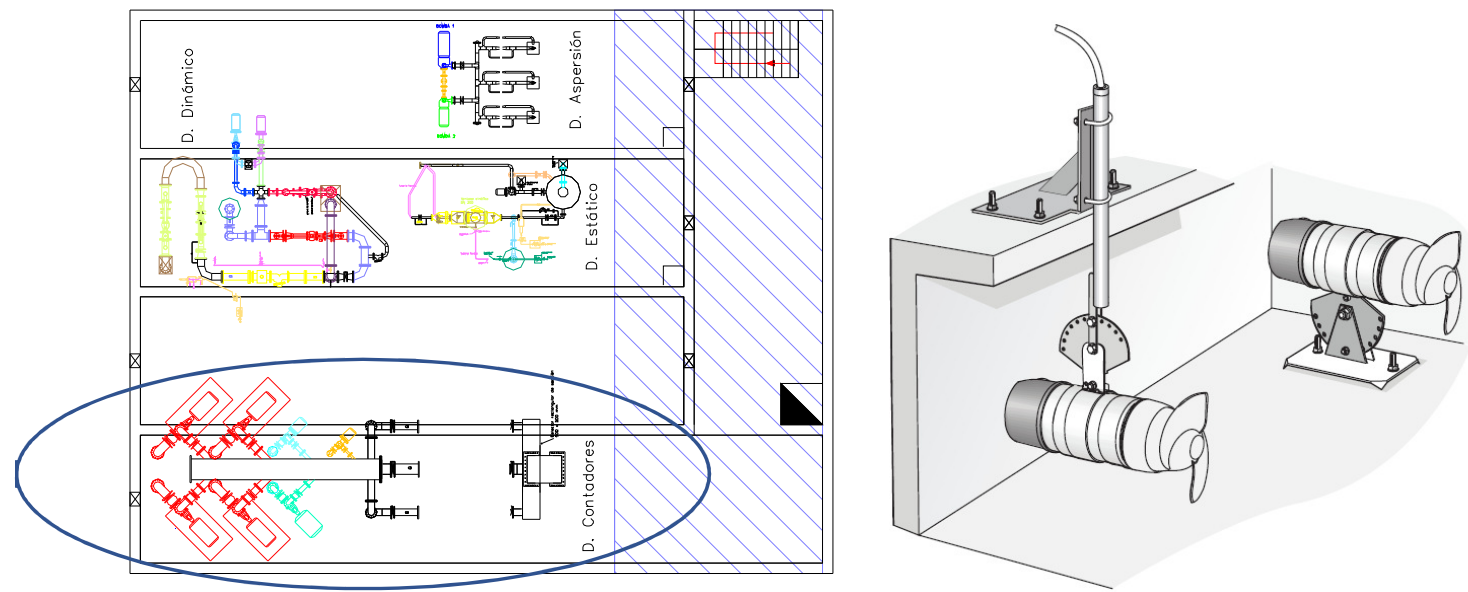

Figura 4: Banco de ensayo de resistencia a las partículas sólidas.

\section{Resultados y discusión}

\subsection{Determinación de la curva de error - caudal}

A continuación, se muestran la curva de error - caudal de una muestra de cada tipo de contador. Las líneas discontinuas rojas indican los errores máximos permitidos (EMP) marcados por la legislación vigente.

CONTADOR WOLTMAN EJE HORIZONAL

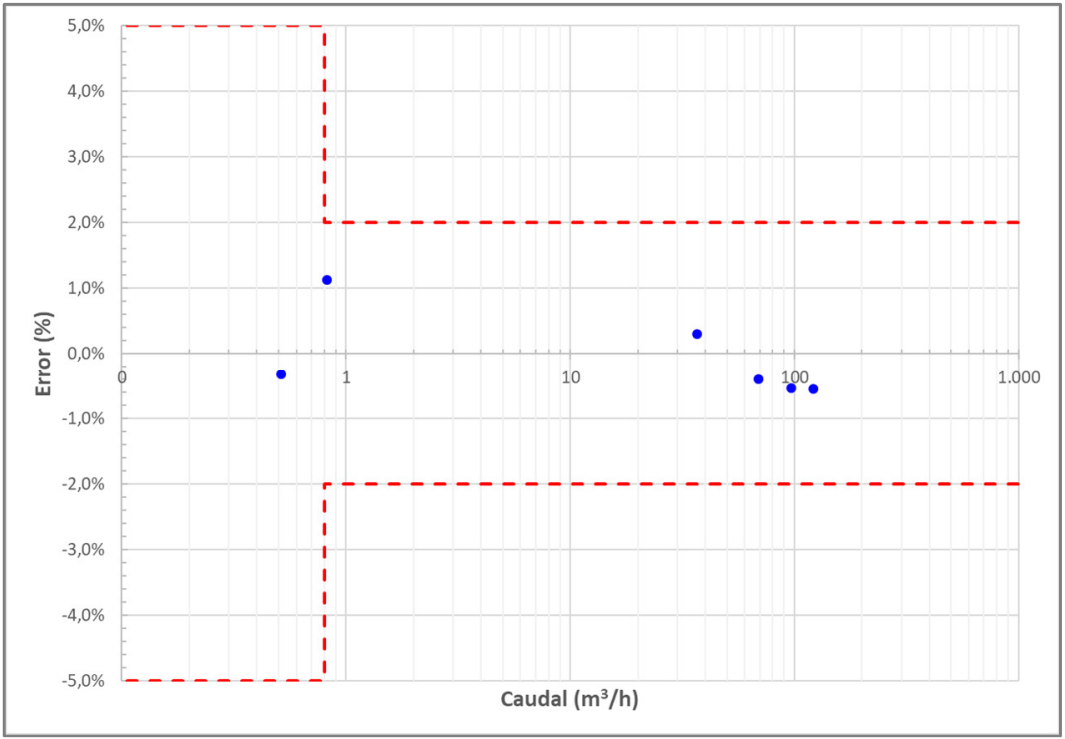

Figura 5: Curva $\mathrm{E}-\mathrm{Q}$ contador $\mathrm{Q}_{3}=100 ; \mathrm{R}=200$. 
CONTADOR WOLTMAN EJE VERTICAL

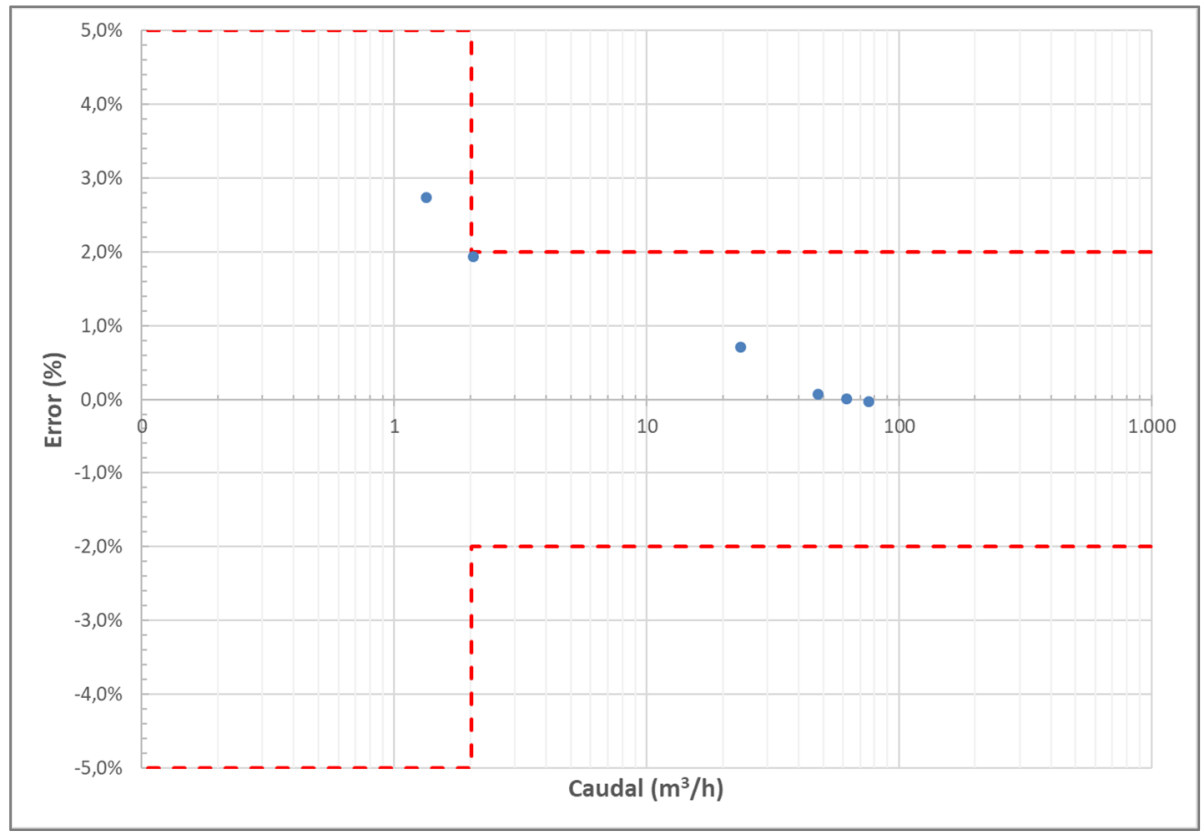

Figura 6: Curva $E-Q$ contador $Q_{3}=63 ; R=50$.

CONTADOR ULTRASONIDOS MODELO 1

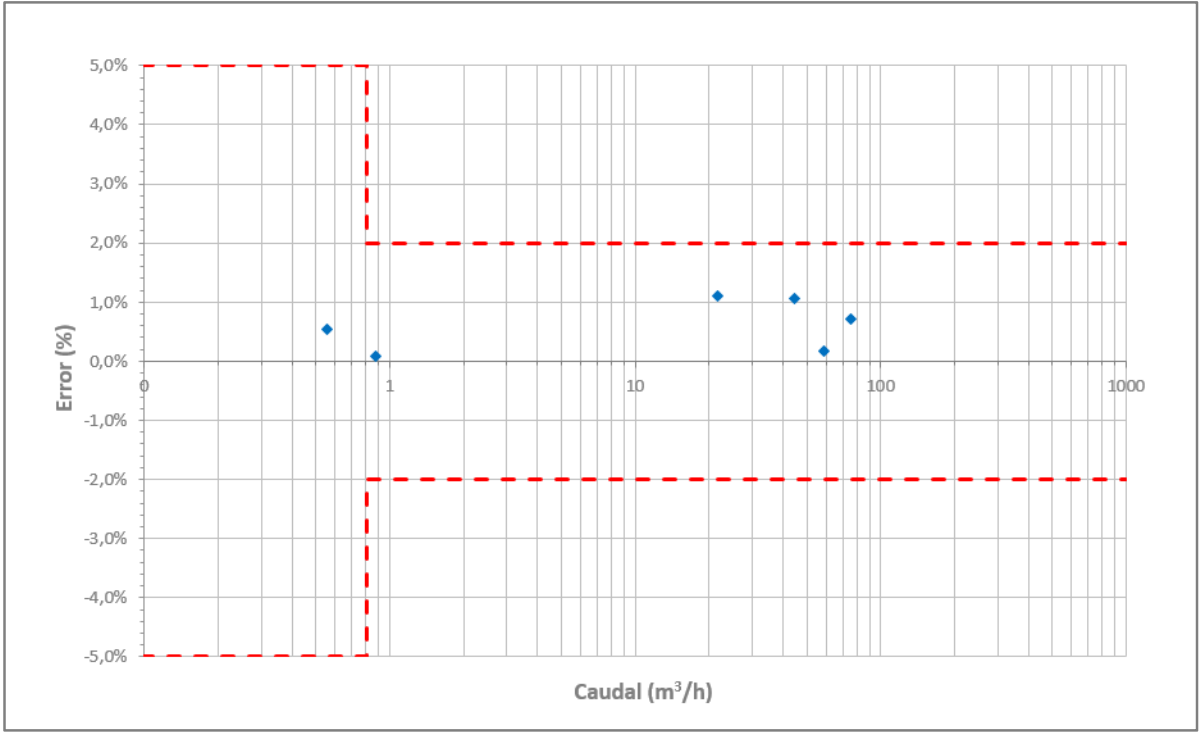

Figura 7: Curva E-Q contador $\mathrm{Q}_{3}=63 ; \mathrm{R}=125$. 


\section{Congreso Nacional de Riegos CARTAGENA 2021

\section{CONTADOR ULTRASONIDOS MODELO 2}

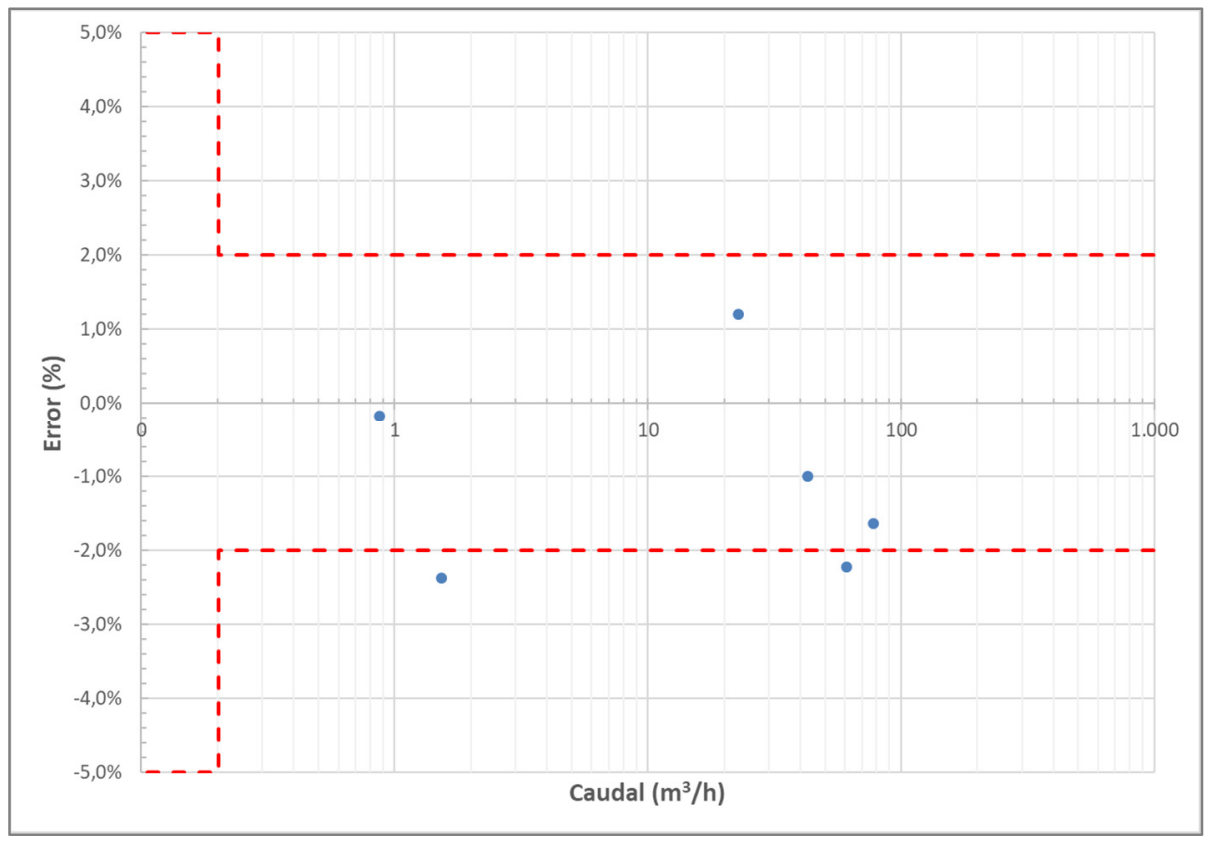

Figura 8: Curva E-Q contador $\mathrm{Q}_{3}=63 ; \mathrm{R}=500$.

En el caso de este equipo, debido a las limitaciones del banco de ensayo del laboratorio del CENTER, no se ha podido determinar el error al caudal mínimo y de transición, haciendo la prueba a un caudal superior. En cualquier caso, con los valores alcanzados en el banco, se puede estimar el comportamiento de este contador y la influencia de las partículas sólidas en su funcionamiento con el paso del tiempo.

Además, se observa que, para algunos de los caudales ensayados, el contador no cumple con los errores máximos permitidos. Sin embargo, no se realiza ningún tipo de ajuste ya que para el objetivo de este trabajo que es comprobar su comportamiento tras el ensayo de resistencia, solo influye la comparativa entre los valores iniciales y finales.

\subsection{Ensayo de resistencia a las partículas sólidas}

A continuación, y en distintas fases, todos los contadores han sido o están siendo sometidos al ensayo de resistencia a las partículas sólidas, realizando controles intermedios para controlar la evolución de la curva de error - caudal con el paso del tiempo.

En el gráfico que se muestra a continuación, correspondiente al contador tipo woltman, se indican en rojo los errores máximos permitidos en origen y, en verde, los errores máximos permitidos después del ensayo de resistencia a las partículas sólidas. 


\section{Congreso Nacional de Riegos CARTAGENA 2021

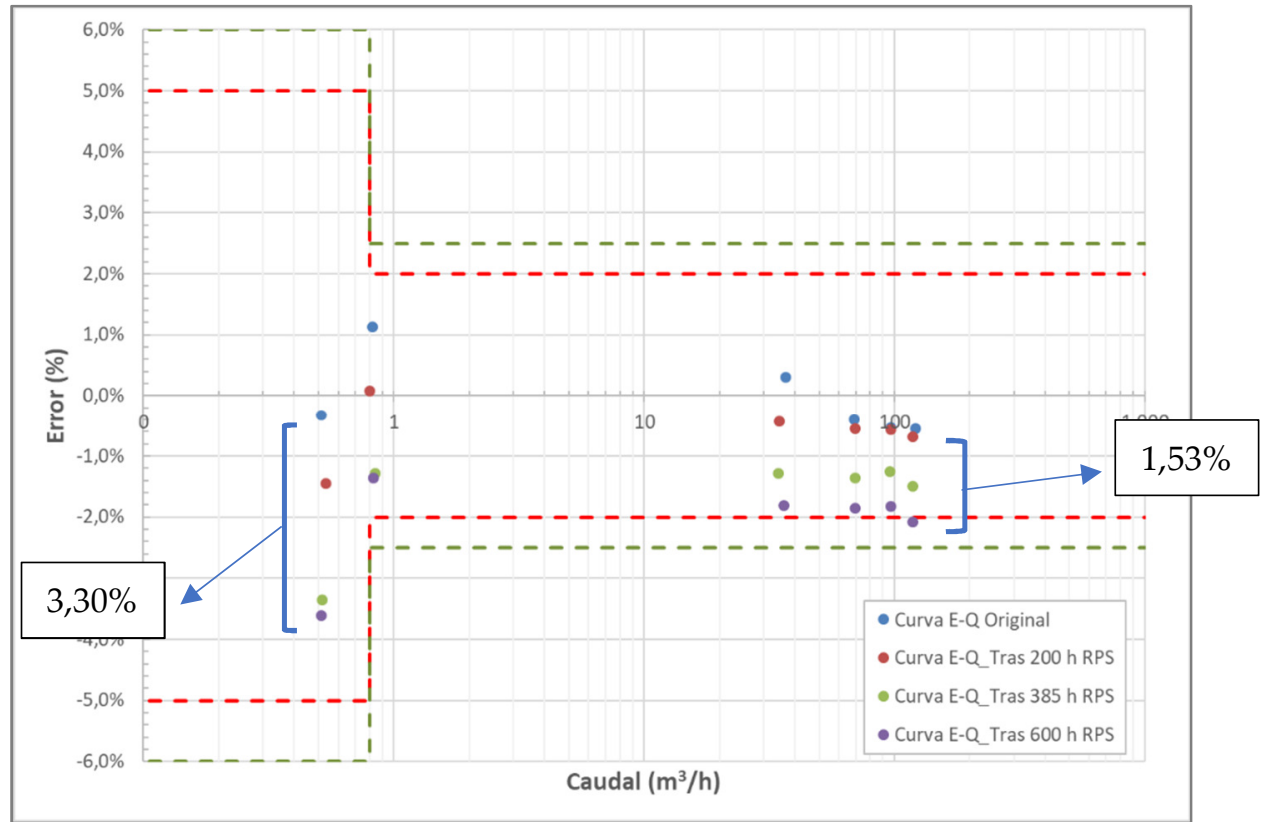

Figura 9: Curva E-Q contador $Q_{3}=100 ; R=200$ (Muestra 1). Comparativa.

Se puede observar que el contador cumple con los errores máximos permitidos, pero no cumple con los requisitos establecidos en cuanto a la variación de la curva de error, sobre todo para los caudales más bajos.

Esto mismo ocurre en las otras muestras de este modelo de contador donde también el condicionante es la variación entre la curva inicial y la curva tras el ensayo de resistencia.

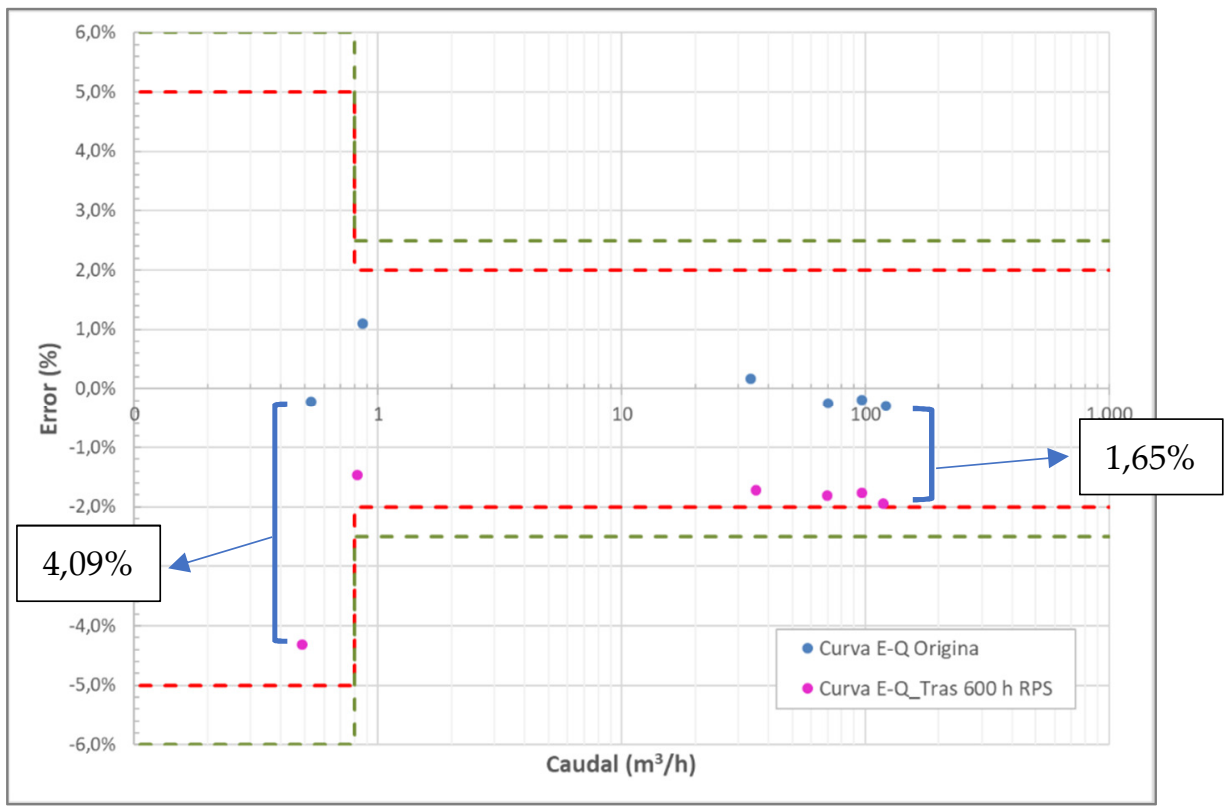

Figura 10: Curva E-Q contador $Q_{3}=100 ; R=200$ (Muestra 2). Comparativa. 
En el momento de redactar esta ponencia, el resto de contadores objeto del estudio están a falta de terminar las $600 \mathrm{~h}$ del ensayo de resistencia a las partículas sólidas por lo que los resultados se mostrarán más adelante.

\section{Conclusiones}

Aunque todavía no se ha podido terminar con todas las pruebas, los resultados obtenidos hasta el momento y la experiencia previa adquirida en el laboratorio del CENTER, permiten sacar las siguientes conclusiones:

1- Los contadores de agua, con el paso del tiempo, tienden al subcontaje, siendo el rango de caudales más bajos el más afectado en las pruebas de durabilidad tanto con agua limpia como con agua con partículas en suspensión.

2- Los rangos de caudal necesarios cuando un contador trabaja como contador de agua limpia son mucho más amplios que cuando es utilizado como contador de riego, ya que la curva de demanda suele ser mucho más variable.

El agua de riego produce mucho más desgaste que el agua limpia y, por tanto, la medición de los caudales bajos se ve afectada más rápidamente, originando que los errores de lectura superen con facilidad los errores máximos permitidos.

Todo ello lleva a plantearse la necesidad de diferenciar los contadores en función de uso.

3- La utilización de contadores en redes de riego que no son mecánicos hacen creer, a priori, que al no tener elementos móviles se verán menos afectados por el desgaste. Sin embargo, algunas tecnologías como los ultrasonidos parece que sí ven modificada considerablemente su curva de error con el paso del tiempo.

Todo esto hace necesario seguir realizando pruebas en el Laboratorio del CENTER con distintos modelos de contadores presentes en el mercado en la actualidad, con el objetivo de conocer su comportamiento y, además, comprobar si los criterios establecidos en la norma ISO 16399 han de ser revisados y adaptados a las nuevas tecnologías. 